\title{
Control of Droplet Evaporation on Oil-Coated Surfaces for the Synthesis of Asymmetric Supraparticles
}

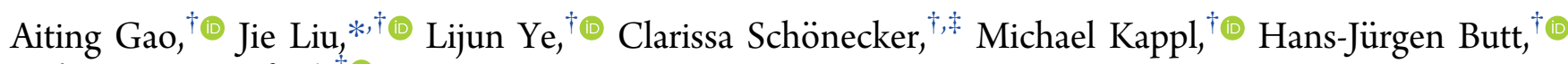 \\ and Werner Steffen* $* \dagger$
}

\author{
${ }^{\dagger}$ Max Planck Institute for Polymer Research, Ackermannweg 10, D-55128 Mainz, Germany \\ ${ }^{\ddagger}$ TU Kaiserslautern, Group for Micro Fluid Mechanics, Gottlieb-Daimler-Straße 49, 67663 Kaiserslautern, Germany
}

Supporting Information

ABSTRACT: Controlling the droplet evaporation on surfaces is desired to get uniform depositions of materials in many applications, for example, in two- and three-dimensional printing and biosensors. To explore a new route to control droplet evaporation on surfaces and produce asymmetric particles, sessile droplets of aqueous dispersions were allowed to evaporate from surfaces coated with oil films. Here, we applied $1-50 \mu \mathrm{m}$ thick films of different silicone oils. Two contact lines were observed during droplet evaporation: an apparent liquidliquid-air contact line and liquid-liquid-solid contact line. Because of the oil meniscus covering part of the rim of the drop, evaporation at the periphery is suppressed. Consequently, the droplet evaporates mainly in the central region of the liquid-air interface rather than at the droplet's edge. Colloidal particles migrate with the generated upward flow inside the droplet and are captured by the receding liquid-air interface. A uniform deposition ultimately forms on the substrate. With this straightforward approach, asymmetric supraparticles have been successfully fabricated independent of particle species.

\section{INTRODUCTION}

Evaporation of water droplets on solid surfaces is a complex process because mass and heat transfer in the liquid and air are coupled. ${ }^{1,2}$ A droplet with suspended colloids ${ }^{2-4}$ or dissolved molecules ${ }^{5-7}$ generally results in ring-like depositions after drying on a solid surface, which is caused by the so-called coffee-ring effect. ${ }^{1,8}$ It is initiated by the pinning of the droplet edge and an evaporation gradient along the droplet surface. Consequently, an outward capillary flow forms inside the evaporating droplet, and the suspended matter transports along with this flow and accumulates at the droplet edge (contact line liquid-air-solid, CLAS). The coffee-ring effect has its applications ${ }^{9}$ for the construction of complex material assemblies in surface patterning, optics, or electronics ${ }^{10-12}$ as well as micro- or nanodevices. ${ }^{13}$ However, the heterogeneity of the deposition greatly restricts its development in inkjetprinting, ${ }^{14}$ photonics, ${ }^{15}$ and biosensors, ${ }^{16}$ where uniform deposition is required.

The final deposition in drying sessile droplets is correlated with the mode of liquid evaporation, ${ }^{17}$ particle adsorption on the liquid-air interface, particle agglomeration, ${ }^{18,19}$ and surface tension gradients along the droplet's surface. ${ }^{20}$ To realize uniform depositions on a substrate, approaches by controlling the evaporation process of droplets have been realized, for example, regulating flow patterns inside evaporating droplets, ${ }^{6,21-23}$ interface deformation, ${ }^{24}$ depinning of CLAS, ${ }^{19,25}$ or modifying the dispersed colloidal particles. ${ }^{26}$ Recently, lubricant-infused surfaces, consisting of micro/nano-structures filled with lubricating liquids, have been discovered with ultralow contact angle hysteresis to foreign immiscible droplets. $^{27-29}$ Yang et al. have released a novel technique with capability of ultrasensitive molecular detection (down to $10^{-15} \mathrm{~mol} \cdot \mathrm{L}^{-1}$ ), which is realized by the enrichment and delivery of analytes into the detective sites based on this platform. ${ }^{30}$ McBride et al. ${ }^{31}$ demonstrated that salt crystallization is suppressed at the droplet edge of salt solution during its evaporation. Das et al. ${ }^{32}$ reported that the coffee-ring effect can be suppressed on a silicone oil-coated surface. In these cases, the lubricant film prevents pinning of the droplet edge at the substrate during droplet evaporation. However, there is a lack of understanding of how the pattern of the deposit is influenced by the droplet, property of the lubricant, suspended particles, and wetting properties of the substrate.

In this work, we investigated how silicone oil films with a thickness of the order of $10 \mu \mathrm{m}$ on a solid surface regulates the evaporation of sessile water droplets and how it suppresses the coffee-ring effect. The mechanisms of particles transport and hence the flow inside of these droplets was studied with $3 \mathrm{D}$ laser confocal microscopy. This evaporation approach was

Received: August 8, 2019

Revised: October 2, 2019

Published: October 7, 2019 
a

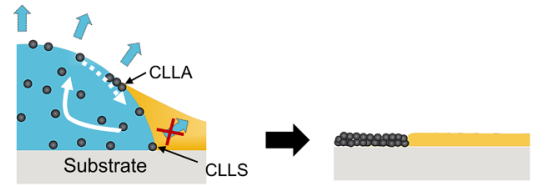

b

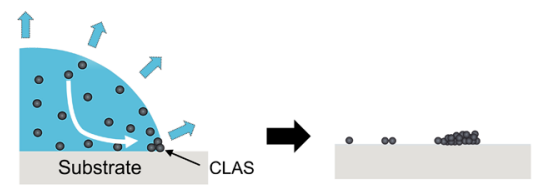

e

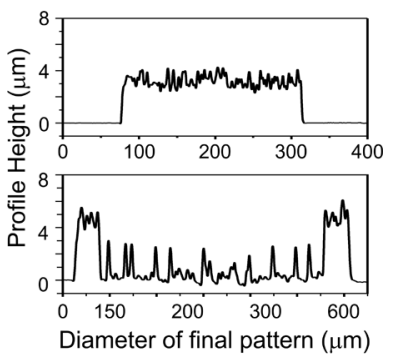

C

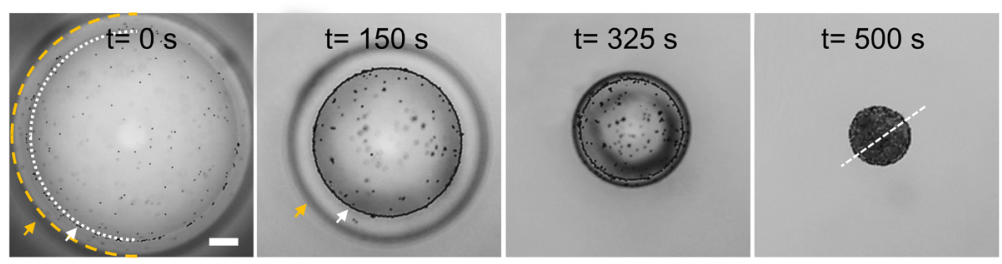

d

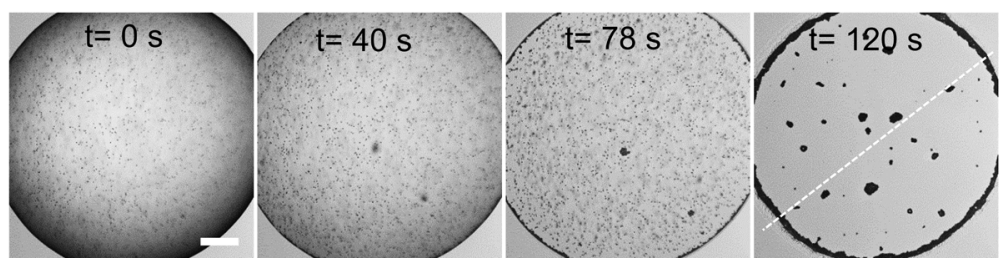

Figure 1. Droplet evaporation. (a,b) Schematics of the evaporation process and deposition on oil-coated surface (a) and solid surface (b). Blue: water droplet; yellow: oil phase; blue arrows mark the evaporation. (c) Top-view images (by optical microscope) of a $0.1 \mu \mathrm{L}$ droplet evaporating on oil-coated hydrophobic glass surface (oil viscosity: $100 \mathrm{cSt}$, thickness: $10 \mu \mathrm{m}$ ). The outer, orange dashed semicircle represents the rim of the droplet base (CLLS). The inner, white dotted semicircle represents the apparent CLLA. The arrows represent the retracting of the corresponding contact lines. (d) Bottom-view images (by inverted confocal microscope) of a $0.1 \mu \mathrm{L}$ droplet evaporating on bare glass surface. Scale bars: $100 \mu \mathrm{m}$. (e) Corresponding height profiles of deposits measured by NanoFocus microscopy along the lines marked with dashed, straight lines in (c,d), respectively.

applied to fabricate disc-like or pill-like asymmetric supraparticles.

\section{MATERIALS AND METHODS}

Silicone oils purchased from Sigma-Aldrich, with viscosities from 10 to $10^{5} \mathrm{cSt}, \gamma \approx 20 \mathrm{mN} / \mathrm{m}$. Two kinds of polystyrene (PS) beads, fluorescently labeled PS (diameter $2.5 \mu \mathrm{m}$, excitable at $630 \mathrm{~nm}$ and emitting in the range of $645-680 \mathrm{~nm}$ ), obtained from life technologies, USA) and unlabeled PS ( $3 \mu \mathrm{m}$, synthesis by dispersion polymerization method in our lab) were used. The vinyl-terminated polydimethylsiloxane (PDMS), cross-linking agent (HMS-301) and platinum catalyst were purchased from Gelest Inc. The wettability of the substrates was measured with a Dataphysics OCA35 goniometer in the sessile drop configuration (DataPhysics Instruments $\mathrm{GmbH}$, Germany). The surface tension measurements were obtained by DataPhysics DCAT11EC tensiometer (PT10, DataPhysics, Germany), with the Wilhelmy plate method. Droplet evaporation was imaged by an inverted laser scanning confocal microscope (Leica TCS SP8 with a $20 \times / 0.75$ multi-immersion objective, $n=1.45$, in the text referred to as confocal microscope) as well as with an optical microscope from above (Carl Zeiss Axiotech Vario 100HD, 10X objective). Deposited patterns and their height profile were imaged by scanning electron microscopy (low voltage LEO 1530 Gemini, Germany, and SU8000, Hitachi, Japan). Height profiles were measured optically with a NanoFocus microscope ( $\mu$ surface, NanoFocus AG, Oberhausen, Germany) via depth profiles of scratches in solid films. In all experiments, the environmental temperature and humidity were $T_{\mathrm{a}}=23 \pm 2{ }^{\circ} \mathrm{C}$ and $\mathrm{RH}=47 \pm 5 \%$.

Preparation of Oil-Coated Surfaces. Glass slides and silicon wafers were used as substrates. They were first ultrasonically cleaned in ethanol, acetone, and isopropanol for $15 \mathrm{~min}$, respectively, and then treated with oxygen plasma (120 W, Diener Electronic Femto) for 10 min. Substrates with different wettability can be obtained with different procedures. Oil-coated hydrophobic substrates were obtained by immediately depositing silicone oil on the substrates after plasma cleaning. The thickness of silicone oil layer $(1-50 \mu \mathrm{m})$ was controlled by adjusting the spin-coating speed of the spin coater. Then, the coated substrates were kept at room temperature for $12 \mathrm{~h}$. Silicone groups in the oil will covalently graft to the substrates and endow the substrates with hydrophobicity $\left(\theta_{\mathrm{a}} \approx 102 \pm 3^{\circ}, \theta_{\mathrm{r}} \approx 92 \pm\right.$ $5^{\circ}$ ). In contrast, by immersing the cleaned substrates in water for 3 hrs after plasma treatment, hydrophilic substrates $\left(\theta_{\mathrm{a}} \approx 75 \pm 3^{\circ}, \theta_{\mathrm{r}} \approx\right.$ $40 \pm 6^{\circ}$ ) were obtained (silicone groups will not graft on the substrate). Then, silicone oil was deposited on top.

Because the thickness of the coated oil film is of the order of $10 \mu \mathrm{m}$ and it is in the liquid state, it is difficult to measure the thickness by conventional profilometry or by optical techniques. Instead, we used a cross-linkable vinyl-terminated PDMS (same viscosity with the silicone oil). Our assumption is, when same spin-coating speed was applied, the thickness of the cross-linked PDMS layer is to be almost the same as liquid silicone oil film. The vinyl-terminated PDMS was mixed with cross-linking agents (4 wt \%) and platinum catalyst (0.005 wt \%) and then spin-coated on the substrates. The coated PDMS layer was cured by placing the substrates in an oven $\left(60^{\circ} \mathrm{C}\right)$ for $8 \mathrm{~h}$. Applying a scratch in the cross-linked PDMS layer, we measured its depth profile with NanoFocus and thus obtained the thickness.

Observation of Particle Transport during the Droplet Evaporation Process. The confocal microscope with a $20 \times / 0.75$ multi-immersion objective was used in these experiments. Fluorescently labeled PS particles $(d=2.5 \mu \mathrm{m}, 0.015 \mathrm{vol} \%$, excited at 561 $\mathrm{nm}$, negatively charged in water) were used as tracers, and the silicone oil was fluorescently labeled with coumarin $6(0.05 \mathrm{mg} / \mathrm{mL}$, excitation wavelength $458-488 \mathrm{~nm}$ ). Microliter-sized droplets of the dispersion $(0.05 \mu \mathrm{L})$ were deposited on the oil-coated substrate. The motions of particles during evaporation were recorded. Refractive indices: silicone oil 1.422; water: 1.33; glass slides: 1.508 . 
a

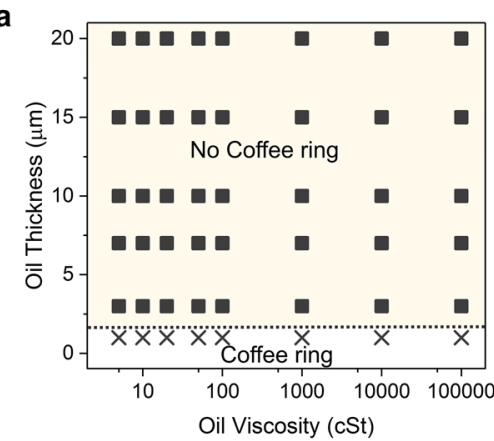

C

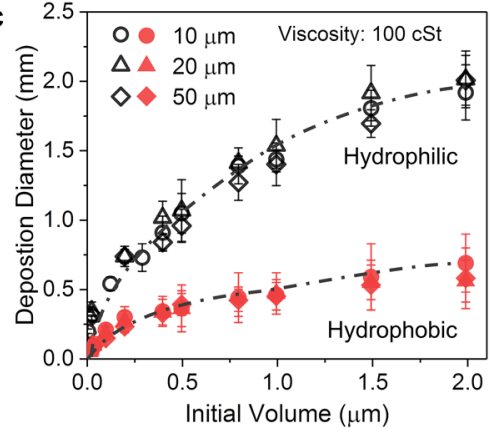

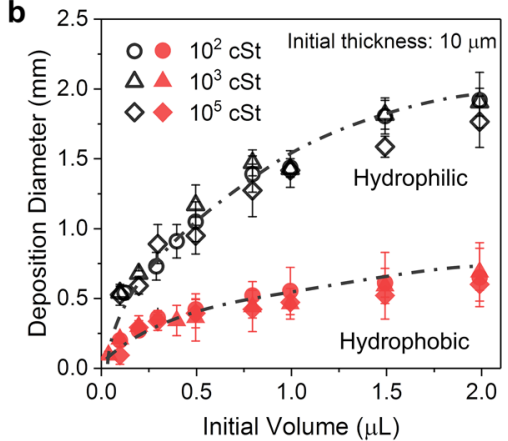

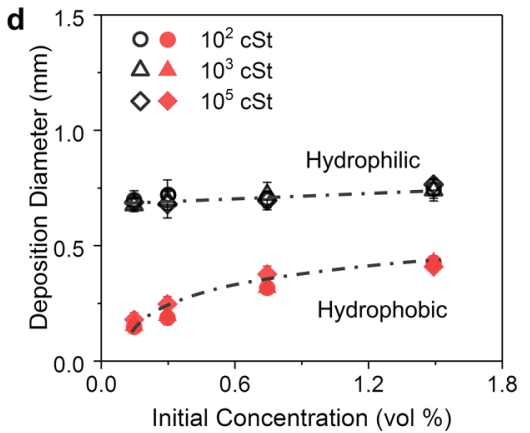

Figure 2. Influence of parameters on the deposited patterns of droplets with PS particle dispersion ( $3 \mu \mathrm{m})$ on oil-coated surfaces. (a) "Phase" diagram of the suppression of coffee ring effect on oil-coated hydrophobic surface, depending on the thickness of oil film and the viscosity of oil. Solid squares $(\square)$ : concentrated deposition; crosses $(x)$ : coffee ring deposition; dotted black line: critical thickness of the oil layer on the surface for suppression of coffee-ring effect. (b,c) Deposition diameter changes with the initial volume of the colloidal droplet, while in (b) oil viscosity and in (c) oil layer thickness varies. (d) Deposition diameter as a function of initial colloidal concentration of droplets.

\section{RESULTS AND DISCUSSION}

Morphology of Droplets Deposited on Oil-Coated Surfaces. When a water droplet is deposited on a silicone oilcoated surface, the oil spontaneously climbs up around the water droplet. ${ }^{33}$ Oil will even wrap over a water droplet if the surface energy difference between oil, water, and air, represented by the spreading coefficient $S_{\mathrm{ow}(\mathrm{a})}=\gamma_{\mathrm{wa}}-\gamma_{\mathrm{wo}}-$ $\gamma_{\mathrm{o} a}$ is positive. ${ }^{34,35}$ In our experiment, the water-air, oil-air and water-oil interfacial tensions are $\gamma_{\mathrm{wa}}=72 \mathrm{mN} / \mathrm{m}, \gamma_{\mathrm{oa}}=20$ $\mathrm{mN} / \mathrm{m}$, and $\gamma_{\text {wo }} \approx 45 \mathrm{mN} / \mathrm{m}$, respectively. Thus, $S_{\text {ow(a) }} \approx 7$ $\mathrm{mN} / \mathrm{m}$, silicone oil will wrap over the droplet. In addition, the oil film thickness $B$ at the top of droplet can be calculated from the balance of disjoining pressure from the van der Waals repulsive force and the capillary force from the curvature of droplet, $B=\left(A_{\mathrm{H}} R / 12 \pi \gamma_{\mathrm{oa}}\right)^{1 / 3} \cdot 36,37\left(A_{\mathrm{H}}\right.$ : Hamaker constant, $A_{\mathrm{H}}$ $\approx 4 \times 10^{-21} \mathrm{~J}, R$ : initial droplet radius). For the droplets with microliters used in this study, the $B$ was calculated to be about $10 \mathrm{~nm}$, which is too thin to be directly experimental observed or to have a significant influence on the evaporation. Therefore, for analytical purposes, we assume the top surface of the droplet is bared and only surrounded by an oil wetting ridge. $^{38}$ In our experiments, we observed an apparent liquidliquid-air contact line (CLLA) on the droplet (Figure 1).

When a water droplet is deposited on an oil-coated surface, the question arises whether or not there is still an oil film underneath this droplet. The stability of a possible oil film underneath this droplet is affected by the wettability of the surface. It has been pointed out ${ }^{28,39}$ that this is determined by the interfacial tensions of oil, colloidal liquid, and solid substrate, which is represented by the spreading constant $S_{\mathrm{ow}(\mathrm{s})}$ $=\gamma_{\mathrm{sw}}-\gamma_{\mathrm{ow}}-\gamma_{\mathrm{os}}=-\gamma_{\mathrm{ow}}\left(\cos \theta_{\mathrm{o}}+1\right)$. Here, $\theta_{\mathrm{o}}$ is the contact angle of the water droplet on the substrate when submerged in a silicone oil-filled bath. On the oil-coated hydrophobic surfaces, we find $\theta_{\mathrm{o}}=177 \pm 3^{\circ} \approx 180^{\circ}$ (Figure S1a), and therefore $S_{\mathrm{ow}(\mathrm{s})}=0$, an oil film is sandwiched between the droplet and the substrate. An interference pattern ${ }^{40}$ originating from the solid-oil and oil-water interfaces, illustrates the existence of a thin oil film underneath the deposited droplet (Figure S1c). In contrast, on the hydrophilic substrates, $\theta_{\mathrm{o}}=$ $135 \pm 5^{\circ}$ (Figure S1b), $S_{\text {ow }(s)} \approx-14 \mathrm{mN} / \mathrm{m}$, the oil film is excluded from the contact area between droplet and substrate. We observe no interference pattern within the resolution of the microscope (Figure S1d), which demonstrates that water is in direct contact with the hydrophilic substrate.

Suppression of the Coffee Ring Effect on the OilCoated Surface. To understand droplet evaporation, the flow of dispersed particles in the droplets, and the shape of deposition formed on the oil-coated surface, we dispersed PS colloids (diameter $3.0 \mu \mathrm{m}, 0.1 \mathrm{vol} \%$ ) in water.

The experimental situation of droplet evaporation on substrates with or without oil films is schematically shown in Figure 1a,b. When a droplet of particle dispersion is deposited on an oil-coated substrate, the oil phase climbs up the droplet. Two apparent contact lines show on the droplet surface: liquid-liquid-air contact line (CLLA) and liquid-liquidsolid contact line (CLLS). This oil ridge hinders the evaporation from the bottom edge (CLLS). Because water loss only occurs at the uncovered top of the droplet, an upward flow generates inside of the evaporating droplet. This alters the particle dispersion during droplet evaporation, yielding a homogenous pattern left on the substrate, which will be discussed later (Figure 1a).

The evolution in time for a $0.1 \mu \mathrm{L}$ droplet of colloidal suspension evaporating from an oil-coated hydrophobic surface is shown in Figure 1c. Two distinct particle-enriched contact lines, the apparent CLLA (inner, white dotted line) and the 
a
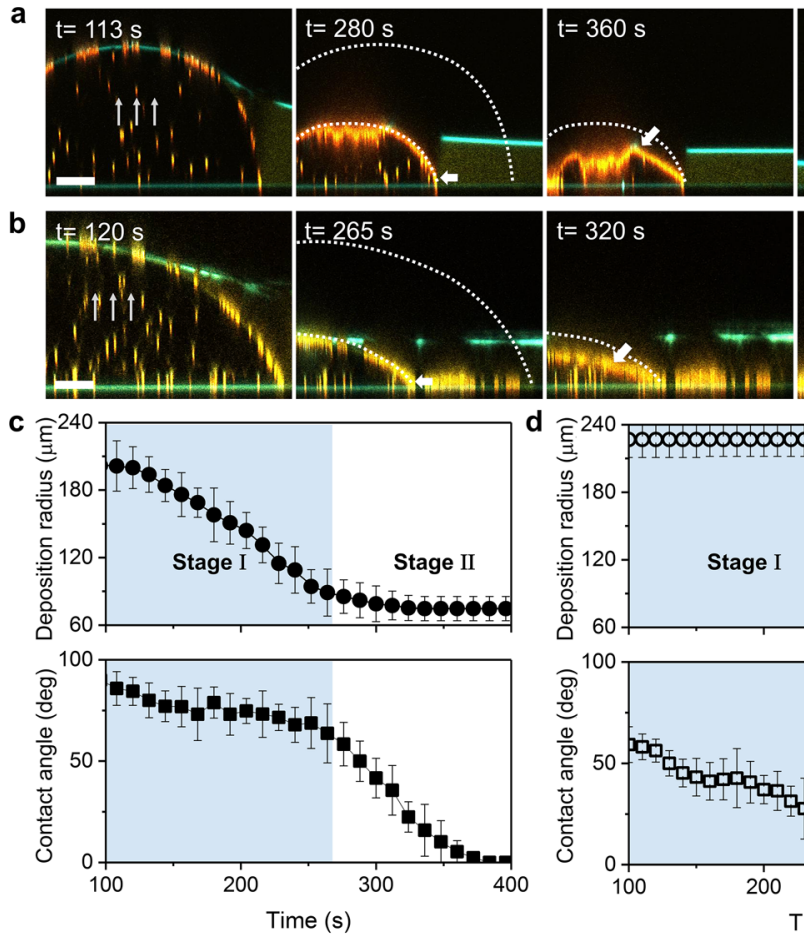

e

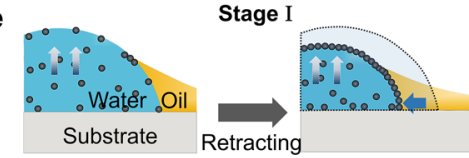

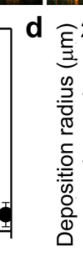
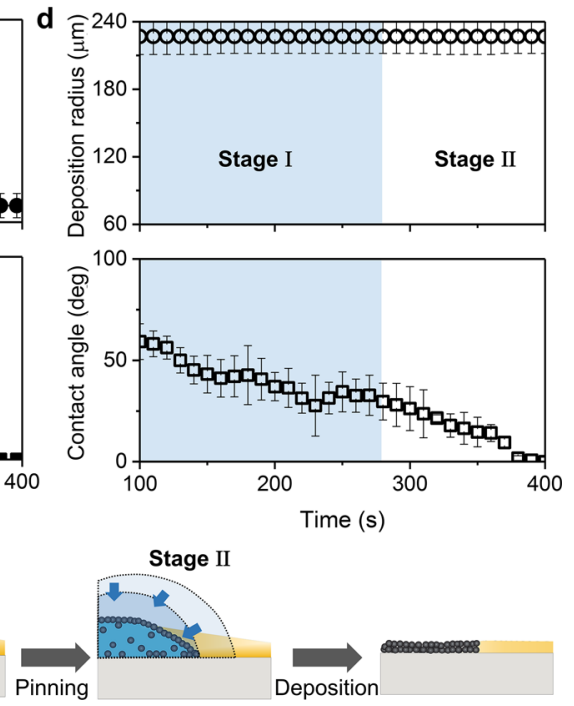

Figure 3. Controlled evaporation of colloidal droplets on oil-coated surfaces. (a,b) Snapshots of colloidal droplets (PS, fluorescent, $2.5 \mu \mathrm{m}$ ) evaporating on oil-coated hydrophobic (a) and hydrophilic (b) surfaces, respectively. The previous and actual profile of the droplets is marked by dotted lines. Parallel arrows: the retracting of the droplet edge (CLLS); tilted arrows: the declining of the droplet profile; upward arrows: upward flow generated inside droplet. Color scheme: orange: fluorescent PS particles; yellow: oil phase; cyan: reflection from interfaces. Scale bars: $50 \mu \mathrm{m}$. $(\mathrm{c}, \mathrm{d})$ Evaporation kinematics of droplets, characterized by deposition base radius $(R)$ and instant contact angle $(\theta)$ on the oil-coated hydrophobic (c) and hydrophilic (d) surfaces. The blue and white background indicate regions of the droplet base line retracting and pinning, respectively. (e) Schematic of the droplet evaporation on the oil-coated surface. Retracting and pinning of the droplet base line is illustrated.

CLLS at the droplet base (outer, orange dashed line), can be observed. Particles tend to transport to the upper interface and enrich there rather than at the bottom edge. This phenomenon indicates the evaporation at the droplet edge is hindered. Particles are more likely to transport to the CLLA, indicating a higher evaporation flux shown near the CLLA contact line (details in Supporting Information). ${ }^{24,41}$ After complete evaporation of the droplet, a uniform deposition is formed on the oil-coated hydrophobic surface.

For comparison, on bare glass surfaces, particles in the droplet continuously transport to the pinning three phase liquid-air-solid contact line (CLAS) because of the outward capillary flow inside the droplet (Figure $1 \mathrm{~b}, \mathrm{~d}$ ). ${ }^{1,3}$ A typical coffee ring forms. We found that the evaporation time is significantly prolonged when droplets evaporate on oil-coated surfaces. It took about $500 \mathrm{~s}$ for a $0.1 \mu \mathrm{L}$ droplet to dry on the oil-coated hydrophobic substrate, whereas it took only $120 \mathrm{~s}$ on a bare glass substrate. The water loss rate in the evaporation process is reduced partly due to the decrease of the liquid-air interface of the droplet and follows the $V(t)^{2 / 3}$ relation for the time of evaporation (eq S7, Figure S2). The corresponding height profiles of the two patterns in Figure le confirm that the oil-coated layer efficiently suppresses the coffee-ring effect, which is independent of the substrate's wetting property (Figure S3).
Influence of Viscosity, Film Thickness, Volume, and Concentration on Depositions. We analyzed the influence of oil viscosity ( 5 to $10^{5} \mathrm{cSt}$ ), film thickness $(1-20 \mu \mathrm{m}$, before droplet deposition on the surface), droplet size $(0.03-2 \mu \mathrm{L})$, and concentration of colloids $(0.015-1.5$ vol \%) as well as substrate wettability on the final structure of depositions (Figure 2).

By depositing $0.2 \mu \mathrm{L}$ of droplets on the oil-coated hydrophobic surface, the "phase" diagram of the efficiency for suppression of the coffee ring is explored (Figure 2a). The coffee-ring effect is suppressed on the oil-coated hydrophobic surface when the initial thickness of the oil is bigger than $1 \mu \mathrm{m}$, irrespective of the oil viscosity. On oil-coated hydrophilic surfaces (Figure S4), the coffee-ring effect could only be suppressed when the initial oil thickness was bigger than $3 \mu \mathrm{m}$. Changing the viscosity there is a slight difference, as the oil thickness can be decreased to $1 \mu \mathrm{m}$ for oils with viscosity $>1000$ cSt.

Within the "No Coffee Ring" region, the diameter of the deposition increased with the increase of the initial droplet volume (Figure 2b). All of the data of the diameter of deposition on oil-coated substrate with different oil viscosities sit on two distinct curves determined only by the wettability of the substrates. The above hydrophilic curve illustrates that larger patterns are formed on oil-coated hydrophilic surfaces. A similar relationship (with same curve corresponding to that in 
Figure 2b) was also observed when the oil layer thickness was changed (Figure 2c). The size of the deposition is determined by the initial size of droplet and wettability of the substrate, while the oil properties (viscosity or thickness) show negligible effects.

The concentration of colloidal suspension was studied (Figure $2 \mathrm{~d}$ ). Droplets $(0.2 \mu \mathrm{L})$ were deposited on oil-coated surfaces, with the varying initial colloid concentrations from 0.015 to $1.5 \mathrm{vol} \%$. On the oil-coated hydrophobic substrate, the final deposition increased with the increase of the colloids concentration and showed concentric patterns (Figure S5a). On the contrary, on hydrophilic substrates, the size of the dried depositions was independent of the initial colloid concentrations and identical with the initial droplet base radius before evaporation occurred. The depositions show larger features (Figure S5b) and indicate that particles deposited on the surface did not move with the retracting droplet edge during droplet evaporation. The deposition difference between the hydrophobic and hydrophilic oil-coated surfaces implies different particle deposition behavior during the evaporation process.

Time Evolution of Droplet Evaporation and Particles Distribution. To gain deeper insight into the flow pattern during evaporation, fluorescent PS particles (diameter $2.5 \mu \mathrm{m}$ ) were added as tracers, and we image the process by a confocal microscope. The dispersion $(0.05 \mu \mathrm{L}, 0.015$ vol \%) was deposited on the oil-coated surfaces and observed by the confocal microscope (Figure 3a,b). On an oil-coated hydrophobic surface (Figure 3a and Video S1), the bottom edge of the droplet continuously retracts with time during the initial evaporation process $(<280 \mathrm{~s})$. No particles were attached to the substrate. During this period, particles are driven to the upper water-air interface and water-oil interface. Through tracing the trajectory of the particle motions inside the evaporating droplet (Figure S6), an upward flow pattern was observed. With evaporation progressing, more and more particles enrich at the water-air interface and temporarily settle at the CLLA. One thing to note, particles enriched at the CLLA might escape and travel downward to the bottom of the droplet along the water-oil interface. When these particles arrived at the bottom droplet edge, they moved inward with the retracting droplet edge. At the last evaporation stage (280$390 \mathrm{~s})$, most of the colloidal particles at the interface are packed into a viscous colloidal skin ${ }^{42}$ and the edge of the droplet began to pin on the substrate. With the continuous loss of water during droplet evaporation, the fully packed skin sinks until it finally deposits on the substrate. A uniform, concentrated pattern ultimately forms on the surface.

The deposition behavior of dispersed particles on oil-coated hydrophilic surfaces was similar (Figure $3 \mathrm{~b}$ ). As the oil-solid interface could be easily replaced by the water-solid interface, ${ }^{33}$ the deposition of particles happened easily and early, which is shown in the image taken at $t=120 \mathrm{~s}$ in Figure $3 \mathrm{~b}$. Images taken from 120 to $320 \mathrm{~s}$ show that some particles attach to the substrate during the depinning of the droplet edge. In this period, dispersed particles in the droplet transport to the upper interfaces. During the last evaporation stage (from 320 to $370 \mathrm{~s}$ ), the edge of the droplet completely pinned on the surface, after which a large and flat deposition is obtained. This evaporation evolution process was confirmed by the $3 \mathrm{D}$ reconstruction of particles motion during droplet evaporation (Figure S7).
The evolution with time of the deposition size $(r)$ and the instant contact angle $(\theta$, at the droplet edge, observed by confocal microscope in xzt mode) is given in Figure $3 c, d$ (corresponding to Figure 3a,b). On oil-coated hydrophobic surfaces (Figure 3c), the deposition radius of droplets with colloidal suspension decreases with time, while the droplet contact angle changes slowly (from 100 to $280 \mathrm{~s}$ ). This reflects the continuous receding of the droplet edge. Once the contact line was pinned on the substrate, the contact angle started to decrease after $t=280 \mathrm{~s}$. On the contrary, the deposition radius did not change on the oil-coated hydrophilic surface (Figure $3 \mathrm{~d})$, indicating the colloids being attached to the substrate at an early stage of the evaporation $(t=120 \mathrm{~s})$. The instant contact angle at the droplet edge was smaller than that on oilcoated hydrophobic surface, and the contact angle continuously decreased in the whole evaporation process.

In conclusion, on oil-coated surfaces drops of dispersions evaporate in two stages (Figure 3e): first, the droplet base line retracts freely. Because water can only evaporate in the central area, which is above the CLLA, there is a central upward flow in the droplet. This flow carries particles to the top. Particles tend to attach at the upper water surface and form a monolayer. In the second stage, the droplet edge is pinned because the upper surface of the droplet has been fully packed with colloids and is hardly to retract anymore. As the volume of the drops keeps shrinking, the layer of particles attached to the water surface is deposited on the solid surface.

Fabrication of Asymmetric Supraparticles. By evaporating droplets of colloidal suspensions on oil-coated hydrophobic surfaces, colloidal supraparticles with various sizes can be obtained. Because a stable thin oil film is formed between the drying droplets and the hydrophobic substrate (Figure S1), the self-assembled supraparticles can be easily rinsed off the substrates by solvents (Figure $4 \mathrm{a}$ ).

Supraparticles of a range of materials were obtained by depositing droplet arrays. The size of the final supraparticles is controlled by the initial volume and concentration of droplets. Subsequently, the obtained supraparticles were collected by immersing the loaded surface in a hexane solution. As examples, the SEM images in Figure $4 b-d$ show supraparticles obtained from droplets with a dispersion of PS $(3 \mu \mathrm{m}, 0.1 \mathrm{vol}$ $\%), \mathrm{TiO}_{2}(25 \mathrm{~nm}, 0.1 \mathrm{vol} \%$ ), and carbon diamond ( $20 \mathrm{~nm}, 0.1$ vol \%), respectively. The shape of the supraparticles varied from a disc-like to a pill-like, to a nearly spherical shape. The different shapes of these supraparticles indicate that dispersed microparticles show an earlier pinning to the substrate as compared to nanoparticles, as a larger jamming area at the liquid-air interface before it pins on the substrate. This is further confirmed by depositing nanoscale PS particle dispersion (68 and $486 \mathrm{~nm} ; 0.1 \mathrm{vol} \%$ ) on the substrate; the dried supraparticles present pill-like shapes (Figure S8). By applying this approach, we can fabricate supraparticles with defined sizes and shapes, which shows no limitation of materials or sizes of the dispersed particles. For instance, pilllike supraparticles are obtained by evaporating $\mathrm{TiO}_{2}, \mathrm{ZnO}$, and $\mathrm{SiO}_{2}$ dispersion solutions as shown in Figure $4 \mathrm{e}-\mathrm{g}$.

\section{CONCLUSIONS}

In this paper, we demonstrate that oil-coated surfaces can be applied to regulate the evaporation of droplets to suppress the coffee-ring effect. With laser scanning confocal microscopy and optical microscopy, we were able to elucidate the mechanism of evaporation. When an aqueous dispersion droplet 


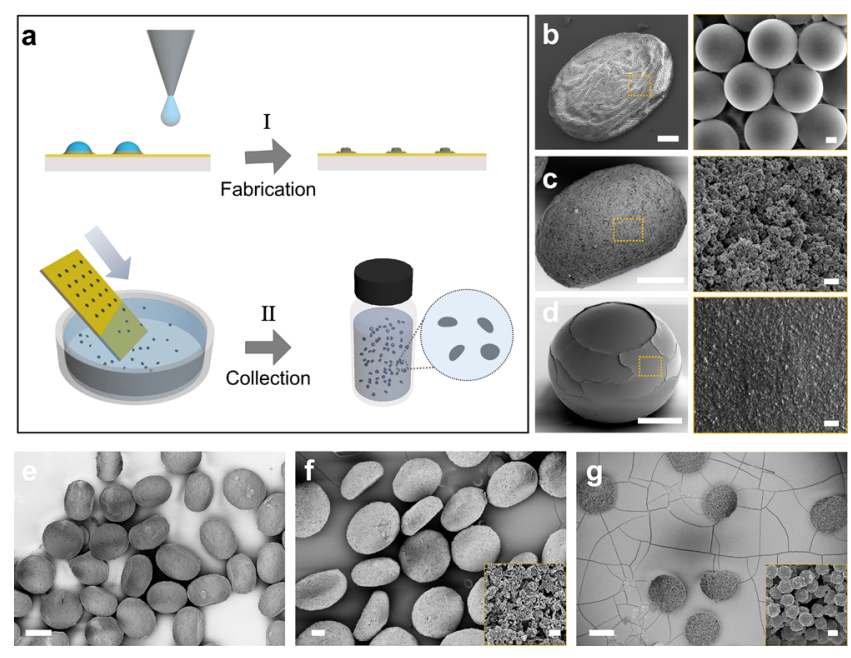

Figure 4. (a) Schematic of the production of asymmetric supraparticles on oil-coated hydrophobic surface and collection process of supraparticles. (b-d) SEM images of the fabricated supraparticles. The scale bars in the left and right: 20 and $1 \mu \mathrm{m}$. (b)A supraparticle formed by evaporating PS solution $(3 \mu \mathrm{m}, 0.1 \mathrm{vol} \%)$ on oil-coated substrate (oil viscosity and thickness: $100 \mathrm{cSt} ; 20 \mu \mathrm{m}$ ). The left image shows the side view of the supraparticle with a disc-like shape. The right image shows the close-up structure. (c) $\mathrm{TiO}_{2}$ supraparticle $(25$ $\mathrm{nm}, 0.1 \mathrm{vol} \%$ ) with a pill-like shape and (d) carbon diamond supraparticle $(20 \mathrm{~nm}, 0.1 \mathrm{vol} \%)$ with a nearly spherical shape. (e-g) Oblate supraparticles obtained by (e) $\mathrm{TiO}_{2}(25 \mathrm{~nm}, 0.1 \mathrm{vol} \%)$, (f) $\mathrm{ZnO}(20 \mathrm{~nm}, 0.1 \mathrm{vol} \%)$ and $(\mathrm{g}) \mathrm{SiO}_{2}(910 \mathrm{~nm}, 0.1 \mathrm{vol} \%)$, respectively. Scale bars: $200 \mu \mathrm{m}$. Insets in (f,g) show the close-up porous structures of the supraparticles. Insets scale bars: $200 \mathrm{~nm}$ and $1 \mu \mathrm{m}$, respectively.

evaporates from solid-supported oil film, the flow in the central region is directed upward. Evaporation at the periphery is hindered by a self-generated oil "wetting ridge" at the droplet edge. Thus, the flow pattern is reversed as compared to evaporation from solid surfaces; on solid surfaces, flow is directed outward toward the rim. The dispersed colloidal particles are driven to the upper part of the droplet and captured by the declining liquid-air interface, resulting the particles deposit at the final stage of evaporation. Uniform deposition patterns were realized in the study by varying oil parameters as thickness of film and viscosity as well as using hydrophilic and hydrophobic substrates. This simple approach can be applied to control the evaporation process of droplets to produce asymmetric supraparticles from micro- to millimeter sizes.

\section{ASSOCIATED CONTENT}

\section{S Supporting Information}

The Supporting Information is available free of charge on the ACS Publications website at DOI: 10.1021/acs.langmuir.9b02464.

Additional experimental details and data (PDF)

Process of evaporation of a droplet on an oil-coated surface observed by $3 \mathrm{D}$ laser scanning confocal microscopy (AVI)

\section{AUTHOR INFORMATION}

\section{Corresponding Authors}

*E-mail: liujie@mpip-mainz.mpg.de (J.L.).

*E-mail: steffen@mpip-mainz.mpg.de (W.S.).
ORCID

Aiting Gao: 0000-0002-2877-6287

Jie Liu: 0000-0002-0014-5635

Lijun Ye: 0000-0002-9760-9263

Michael Kappl: 0000-0001-7335-1707

Hans-Jürgen Butt: 0000-0001-5391-2618

Werner Steffen: 0000-0001-6540-0660

\section{Notes}

The authors declare no competing financial interest.

\section{ACKNOWLEDGMENTS}

This work was supported by ERC for the Advanced Grant 340391-SuPro (H.-J.B.). A.G. is sponsored by the China Scholarship Council. Y. Cang, D. G. Gonzalez, C. H. Tu, M. Mueller, W. D. Liu and P. Verding are acknowledged for technical support and discussions.

\section{REFERENCES}

(1) Deegan, R. D.; Bakajin, O.; Dupont, T. F.; Huber, G.; Nagel, S. R.; Witten, T. A. Capillary flow as the cause of ring stains from dried liquid drops. Nature 1997, 389, 827.

(2) Deegan, R. D.; Bakajin, O.; Dupont, T. F.; Huber, G.; Nagel, S. R.; Witten, T. A. Contact line deposits in an evaporating drop. Phys. Rev. E 2000, 62, 756-765.

(3) Deegan, R. D. Pattern formation in drying drops. Phys. Rev. E 2000, 61, 475-485.

(4) Bigioni, T. P.; Lin, X. M.; Nguyen, T. T.; Corwin, E. I.; Witten, T. A.; Jaeger, H. M. Kinetically driven self assembly of highly ordered nanoparticle monolayers. Nat. Mater. 2006, 5, 265-270.

(5) Kaya, D.; Belyi, V. A.; Muthukumar, M. Pattern formation in drying droplets of polyelectrolyte and salt. J. Chem. Phys. 2010, 133, 114905.

(6) Sempels, W.; De Dier, R.; Mizuno, H.; Hofkens, J.; Vermant, J. Auto-production of biosurfactants reverses the coffee ring effect in a bacterial system. Nat. Commun. 2013, 4, 1757.

(7) Andac, T.; Weigmann, P.; Velu, S. K. P.; Pinçe, E.; Volpe, G.; Volpe, G.; Callegari, A. Active matter alters the growth dynamics of coffee rings. Soft Matter 2019, 15, 1488-1496.

(8) Nadkarni, G. D.; Garoff, S. An investigation of microscopic aspects of contact angle hysteresis: Pinning of the contact line on a single defect. Europhys. Lett. 1992, 20, 523.

(9) Kuang, M.; Wang, L.; Song, Y. Controllable printing droplets for high-resolution patterns. Adv. Mater. 2014, 26, 6950-6958.

(10) Layani, M.; Gruchko, M.; Milo, O.; Balberg, I.; Azulay, D.; Magdassi, S. Transparent conductive coatings by printing coffee ring arrays obtained at room temperature. ACS Nano 2009, 3, 3537-3542.

(11) Li, S.; Chun, Y. T.; Zhao, S.; Ahn, H.; Ahn, D.; Sohn, J. I.; Xu, Y. B.; Shrestha, P.; Pivnenko, M.; Chu, D. P. High-resolution patterning of solution-processable materials via externally engineered pinning of capillary bridges. Nat. Commun. 2018, 9, 393.

(12) Su, M.; Li, F.; Chen, S.; Huang, Z.; Qin, M.; Li, W.; Zhang, X.; Song, Y. Nanoparticle Based Curve Arrays for Multirecognition Flexible Electronics. Adv. Mater. 2016, 28, 1369-1374.

(13) Zhang, B.; Fanshu, M.; Jiangang, F.; Jingxia, W.; Yuchen, W.; Lei, J. Manipulation of Colloidal Particles in Three Dimensions via Microfluid Engineering. Adv. Mater. 2018, 30, 1707291.

(14) Park, J.; Moon, J. Control of colloidal particle deposit patterns within picoliter droplets ejected by ink-jet printing. Langmuir 2006, $22,3506-3513$.

(15) Kuang, M.; Wang, J.; Bao, B.; Li, F.; Wang, L.; Jiang, L.; Song, Y. Inkjet Printing Patterned Photonic Crystal Domes for Wide Viewing-Angle Displays by Controlling the Sliding Three Phase Contact Line. Adv. Opt. Mater. 2014, 2, 34-38.

(16) Dugas, V.; Broutin, J.; Souteyrand, E. Droplet evaporation study applied to DNA chip manufacturing. Langmuir 2005, 21, 91309136. 
(17) Fischer, B. J. Particle convection in an evaporating colloidal droplet. Langmuir 2002, 18, 60-67.

(18) Anyfantakis, M.; Geng, Z.; Morel, M.; Rudiuk, S.; Baigl, D. Modulation of the coffee-ring effect in particle/surfactant mixtures: the importance of particle-interface interactions. Langmuir 2015, 31, $4113-4120$.

(19) Li, Y.; Yang, Q.; Li, M.; Song, Y. Rate-dependent interface capture beyond the coffee-ring effect. Sci. Rep. 2016, 6, 24628.

(20) Crivoi, A.; Duan, F. Elimination of the coffee-ring effect by promoting particle adsorption and long-range interaction. Langmuir 2013, 29, 12067-12074.

(21) Still, T.; Yunker, P. J.; Yodh, A. G. Surfactant-Induced Marangoni Eddies Alter the Coffee-Rings of Evaporating Colloidal Drops. Langmuir 2012, 28, 4984-4988.

(22) Boulogne, F.; Ingremeau, F.; Dervaux, J.; Limat, L.; Stone, H. A. Homogeneous deposition of particles by absorption on hydrogels. Europhys. Lett. 2015, 112, 48004.

(23) Malinowski, R.; Volpe, G.; Parkin, I. P.; Volpe, G. Dynamic Control of Particle Deposition in Evaporating Droplets by an External Point Source of Vapor. J. Phys. Chem. Lett. 2018, 9, 659-664.

(24) Yunker, P. J.; Still, T.; Lohr, M. A.; Yodh, A. G. Suppression of the coffee-ring effect by shape-dependent capillary interactions. Nature 2011, 476, 308-311.

(25) Cui, L.; Zhang, J.; Zhang, X.; Li, Y.; Wang, Z.; Gao, H.; Wang, T.; Zhu, S.; Yu, H.; Yang, B. Avoiding coffee ring structure based on hydrophobic silicon pillar arrays during single-drop evaporation. Soft Matter 2012, 8, 10448-10456.

(26) Mampallil, D.; Eral, H. B. A review on suppression and utilization of the coffee-ring effect. Adv. Colloid Interface Sci. 2018, $252,38-54$.

(27) Wong, T.-S.; Kang, S. H.; Tang, S. K. Y.; Smythe, E. J.; Hatton, B. D.; Grinthal, A.; Aizenberg, J. Bioinspired self-repairing slippery surfaces with pressure-stable omniphobicity. Nature 2011, 477, 443447.

(28) Lafuma, A.; Quéré, D. Slippery pre-suffused surfaces. Europhys. Lett. 2011, 96, 56001.

(29) Quéré, D. Non-sticking drops. Rep. Prog. Phys. 2005, 68, 24952532.

(30) Yang, S.; Dai, X.; Stogin, B. B.; Wong, T.-S. Ultrasensitive surface-enhanced Raman scattering detection in common fluids. Proc. Natl. Acad. Sci. U.S.A. 2016, 113, 268-273.

(31) McBride, S. A.; Dash, S.; Varanasi, K. K. Evaporative Crystallization in Drops on Superhydrophobic and Liquid-Impregnated Surfaces. Langmuir 2018, 34, 12350-12358.

(32) Das, S.; Dey, A.; Reddy, G.; Sarma, D. D. Suppression of the Coffee-Ring Effect and Evaporation-Driven Disorder to Order Transition in Colloidal Droplets. J. Phys. Chem. Lett. 2017, 8, 4704-4709.

(33) Smith, J. D.; Dhiman, R.; Anand, S.; Reza-Garduno, E.; Cohen, R. E.; McKinley, G. H.; Varanasi, K. K. Droplet mobility on lubricantimpregnated surfaces. Soft Matter 2013, 9, 1772-1780.

(34) Mistura, G.; Pierno, M. Drop mobility on chemically heterogeneous and lubricant-impregnated surfaces. Adv. Phys. 2017, 2, 591-607.

(35) Tan, H.; Diddens, C.; Versluis, M.; Butt, H.-J.; Lohse, D.; Zhang, X. Self-wrapping of an ouzo drop induced by evaporation on a superamphiphobic surface. Soft Matter 2017, 13, 2749-2759.

(36) Schellenberger, F.; Xie, J.; Encinas, N.; Hardy, A.; Klapper, M.; Papadopoulos, P.; Butt, H.-J.; Vollmer, D. Direct observation of drops on slippery lubricant-infused surfaces. Soft Matter 2015, 11, 76177626.

(37) Butt, H.-J.; Kappl, M. Surface and Interfacial Forces; Wiley Online Library, 2010.

(38) Guan, J. H.; Wells, G. G.; Xu, B.; McHale, G.; Wood, D.; Martin, J.; Stuart-Cole, S. Evaporation of Sessile Droplets on Slippery Liquid-Infused Porous Surfaces (SLIPS). Langmuir 2015, 31, 1178111789.

(39) Baker, H. R.; Bascom, W. D.; Singleterry, C. R. The adhesion of ice to lubricated surfaces. J. Colloid Sci. 1962, 17, 477-491.
(40) Daniel, D.; Timonen, J. V. I.; Li, R.; Velling, S. J.; Aizenberg, J. Oleoplaning droplets on lubricated surfaces. Nat. Phys. 2017, 13, $1020-1025$.

(41) Gelderblom, H.; Bloemen, O.; Snoeijer, J. H. Stokes flow near the contact line of an evaporating drop. J. Fluid Mech. 2012, 709, 6984.

(42) Du, K.; Glogowski, E.; Emrick, T.; Russell, T. P.; Dinsmore, A. D. Adsorption energy of nano- and microparticles at liquid-liquid interfaces. Langmuir 2010, 26, 12518-12522. 